\title{
New control approaches to improve contact quality in the conventional spans and overlap section in a high-speed catenary system
}

Proc IMechE Part F:

J Rail and Rapid Transit

2019, Vol. 233(9) 988-999

(C) IMechE 2019

Article reuse guidelines:

sagepub.com/journals-permissions

DOI: | $0.1|77 / 09544097| 882286 \mid$

journals.sagepub.com/home/pif

@SAGE

\author{
Farzad Vesali', Habibollah Molatefi' (D), Mohammad A Rezvani', \\ Bijan Moaveni ${ }^{2}$ and Markus Hecht $^{3}$
}

\begin{abstract}
Continuous and quality contact between the pantograph and the catenary system is one of the major challenges for increasing the speed of electric trains. Compared to other studies, this paper has considered the catenary system as the main system and has assumed the pantograph as an excitation factor. Based on this, a controller has been applied on the contact wire, once at the last span and another time near the contact point of the pantograph. Results were compared with the conventional controllers that exert control force on a collector's head. Based on this, two different objectives were considered for the controller including 'improvement of contact quality' and 'minimisation of the vertical velocity of the overlap point'. For this purpose, a full analytical model of the catenary system was presented and was verified using the relevant standards, and then three types of linear quadratic optimal controllers were added to the model with the two objectives mentioned above. The results of the study show that if the model aims to reduce the overlap point vertical velocity, contact quality will be improved. However, in case it aims to enhance contact quality, the velocity of the overlap point will not necessarily be reduced. Moreover, the contact point controller aiming at reducing the overlap point velocity outperforms other controllers and makes 71\% improvement in contact quality in comparison with the no-controller case.
\end{abstract}

\section{Keywords}

Linear quadratic regulator, contact wire, catenary system, analytical model, pantograph, contact quality, overlap section

Date received: 14 August 2018; accepted: 9 December 2018

\section{Introduction}

Today, all of the world's high-speed fleets are electric, as diesel locomotives are essentially unable to generate power to reach high speeds. ${ }^{1}$ As contact force oscillations amplify at high speeds, which may lead to mechanical wear or the electrical wear of pantograph collector head and/or contact wire, the dynamic interaction of pantograph and catenary system is one of the most critical limiting factors at the maximum speed of a fleet. ${ }^{2}$ The pantograph is a device over the roof of locomotive collecting power from catenary. A catenary system usually consists of two horizontal wires (a messenger cable and a contact wire) and many vertical cables (droppers). The contact wire is in contact with the pantograph and the messenger cable bears contact wire weight using droppers and prevents them from sagging. Figure 1 shows a diagram of the pantograph and catenary system. The most important function of the pantograph is to maintain the contact force of the collector's head by changing the height of the contact wire. It is favourable to reduce dynamic force oscillations in the interaction of the pantograph and catenary system, which is called contact quality. To study the effect of different parameters on contact quality, various software or models have been developed. Some of them use FEM and have made a 3D model of catenary, ${ }^{3}$ some others use FD for $2 \mathrm{D}$ model of catenary ${ }^{4}$ and Seo et al. ${ }^{5}$ have generalised the catenary model to large deformation analysis.

\footnotetext{
'School of Railway Engineering, Iran University of Science and Technology, Tehran, Iran

${ }^{2}$ Faculty of Electrical Engineering, K. N. Toosi University of Technology, Tehran, Iran

${ }^{3}$ Department of Rail Vehicle, Technical University of Berlin, Berlin, Germany
}

Corresponding author: Habibollah Molatefi, Iran University of Science and Technology Narmak, Tehran 16846I3114, Iran. Email: molatefi@iust.ac.ir 


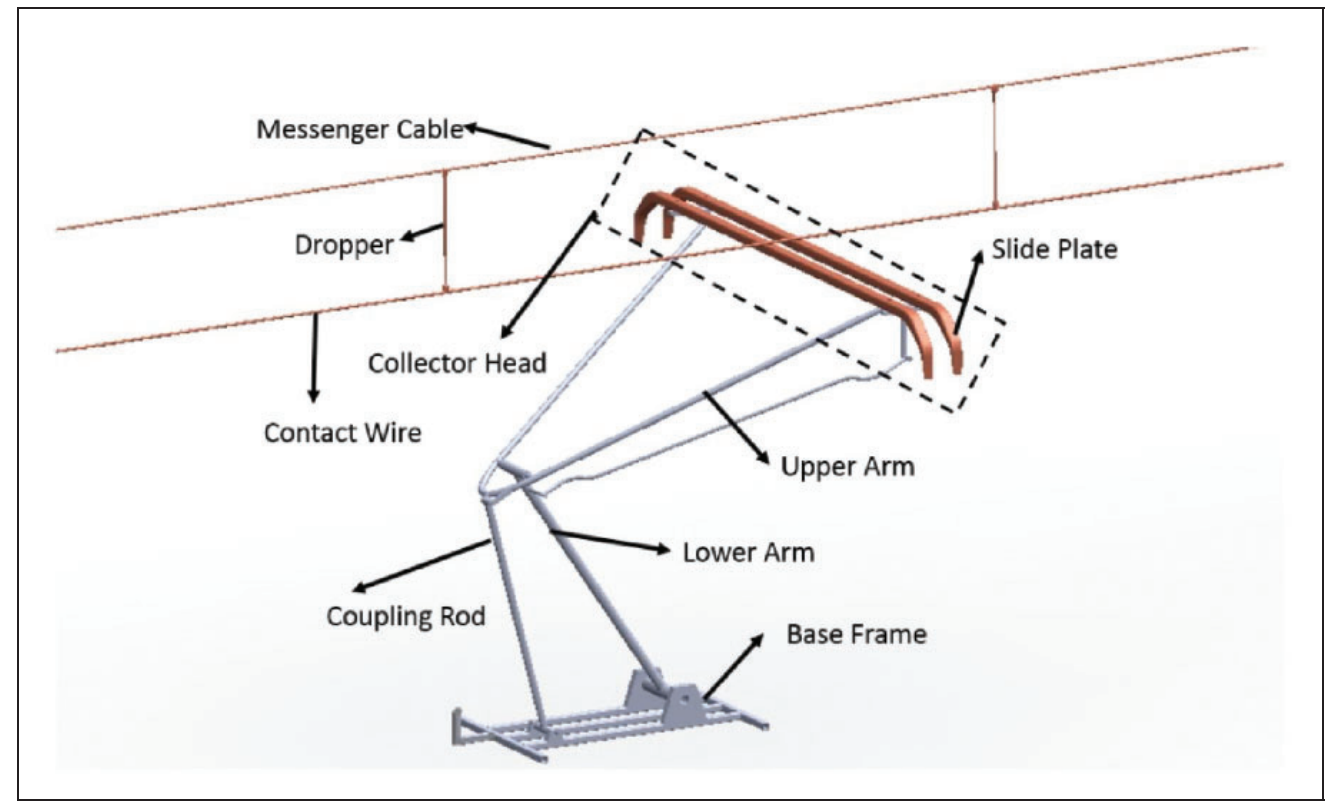

Figure I. Components of the pantograph and catenary system.

The primary challenge is to improve the contact quality, in particular for high-speed trains either by active controlling or optimised design of the pantograph. There are many studies on the optimisation of geometric and dynamic characteristics of pantographs. ${ }^{6,7}$ In the next step, some researchers followed the use of an active controller in a pantograph. ${ }^{8-12}$ In 2005-2015, many researchers concentrated on the pantographs with active controllers; however, the studies had insufficient outputs in the industry, and few of them reached the prototyping step,$^{13}$ as they provided no considerable improvement in contact quality as compared with the increase of initial cost. The relative failure in the performance of pantographs with an active controller allowed the problem to be solved in this study using a change in attitude. In all the reviews on the use of an active controller, a pantograph has been introduced as the main system and contact wire in a catenary system as an excitation factor, but this study considers a catenary system as the main system whose oscillations should be controlled. A pantograph has been considered as the factor causing oscillations. Therefore, the control force has been applied to the contact wire for the first time in this study.

The contact quality between the pantograph and catenary system along the whole route is not fixed and it is lower at some points, which are called 'critical points' in Harell et al. ${ }^{14}$ Contact wire replacement place is one of these points (overlap section). The tensioning device, ${ }^{15}$ which commonly consists of a wheel and some suspended weights, is used to make the expansion and contraction of the contact wire and messenger cable has no impact on its tension. It is designed for a limited length of contact wire (about $1200-1500 \mathrm{~m}$ ). After that, there should be another contact wire with an independent tension system. The overlap section of contact wires is recognised as a critical point, and one of the bottlenecks of contact quality is between the pantograph and catenary system. In the static analysis of the catenary system, a parameter is defined as 'elasticity'. Elasticity means the vertical deflection of the contact wire against a unit vertical load. Presence of droppers and masts in the catenary system makes the elasticity of the catenary system variable. Elasticity variation is one of the most important reasons for oscillations in contact force. In Harell et al., ${ }^{14}$ the researcher introduces the section overlaps and section insulator as the critical section of the catenary-pantograph system based on experimental results and interviews with personnel at Swedish National Administration. Elasticity for catenary is defined as the displacement caused by the vertical unit force. In the overlap section, the applied load must raise the contact wire of both sections. Therefore, a considerable reduction of elasticity occurs at the overlap point; for this reason, this point is considered as a bottleneck. It is clear that contact quality improvement should be at bottleneck points to lead to the overall increase of course speed. Based on this, the controller in this study aims at improving contact quality and reducing impact in the overlap section of the contact wire (cost function).

It can be mentioned that this study considered two new issues. The controller aimed at reducing contact force oscillations and reducing impact at the overlap section of the wire. The control force was once imposed on the pantograph and another time on the catenary system and the results were compared.

Standard EN 50318 has introduced a reference model (pantograph and catenary) and has given all required parameters, to be considered for simulation by any software. Then the standard defined seven parameters for comparing and validating the results of software, which include mean values of contact force, 
the standard deviation of contact force, the statistical maximum of contact force, the statistical minimum of contact force, the actual maximum of contact force, the actual minimum of contact force and the maximum uplift at support. In the next step, the software should be validated via the results of the field test. The considered software for this study is verified by EN 50318 standard.

In the following section, the controller was imposed on a tensile beam to study its performance. Then, three types of actuators were considered on the complete model of catenary system and pantograph, one on the collector head, other on the last span of the catenary system near the overlap span and the last one on the catenary system and near the contact point with the pantograph. Two separate cost functions were considered including contact force oscillations and overlap point vertical velocity. Therefore, six control problems were generally considered and the results were compared.

\section{Analytical model}

Concerning the complexity and variety of pantograph and catenary system components, the models formulated for the analysis of the dynamic interaction of pantograph and catenary system are considered as software. A variety of software are available all over the world for this task, and Bruni et al. ${ }^{16}$ compared them in 2015. The software, which was formulated in Iran University of Science and Technology, is known as CatAna whose major unique feature is the analytical solution. In other words, it never uses the finite element methods and calculates catenary system motion using the Galerkin method and eigenfunction expansion. Two options are available for modelling the pantograph: multibody model and low-order model. ${ }^{17}$ A multibody model involves many details such as kinematic constraints of joints and geometric features of components, while the low-order model considers equivalent mass, stiffness and damping of each degree of freedom. Low-order models are commonly used with numerator translation degree of freedom. Following the reference model of EN 50318, the pantograph was considered as a 2-DOF system. The contact wire and messenger cable were modelled in CatAna as a tensile Euler-Bernoulli beam; the droppers and supports were considered as the springs with a specified stiffness and the mass of clamps of droppers was considered. In the leading software, the droppers are subject to buckling at compression and bear no force; however, this study overlooked the non-linear behaviour of droppers (buckling) concerning the use of the linear controller. Figure 2 shows a schematic diagram of the analytical model.

Equation (1) expresses the governing equations for contact wire and messenger cable

$$
\begin{aligned}
& \rho A w_{, t t}+C w_{, t}-P w_{, x x}+E I w_{, x x x x} \\
& =F_{d r}\left(x_{d r}, t\right)+F_{s}\left(x_{s}, t\right) \\
& \quad+F_{p}\left(x_{p}, t\right)+F_{c}\left(x_{c}, t\right), \quad 0 \leqslant x \leqslant l, \quad t \geqslant 0
\end{aligned}
$$

where $\rho A$ is the mass per unit length, $C$ is the damping of each wire, $P$ is the tension applied to the wire and $E I$ is the bending stiffness of wire. $F_{p}$ is the force imposed by the pantograph; $x_{p}$ is the place to use the force, which is obtained by multiplying velocity by time as the pantograph velocity is fixed; and $F_{c}$ is the control force applied to the wire in position $x_{\text {cont }}$. $F_{s}$ and $F_{d r}$ are the forces of supports and droppers; each is applied to its relevant position $\left(x_{s}\right.$ and $x_{d r}$ ). $w(x, t)$ is the vertical displacement of the wire at the location of $x$ and time of $t$. $t$ and $x$ subscripts, respectively, indicate the partial differentiation from time and displacement. Based on the variation separation method, the answer to the differential equation of equation (1) can be considered as $w(x, t)=X(x) T(t)$. The natural frequencies and mode shapes of the contact wire and the messenger cable can be calculated by inserting the answer in the homogeneous equation proportional to equation (1) and concerning the boundary condition and relevant initial conditions.

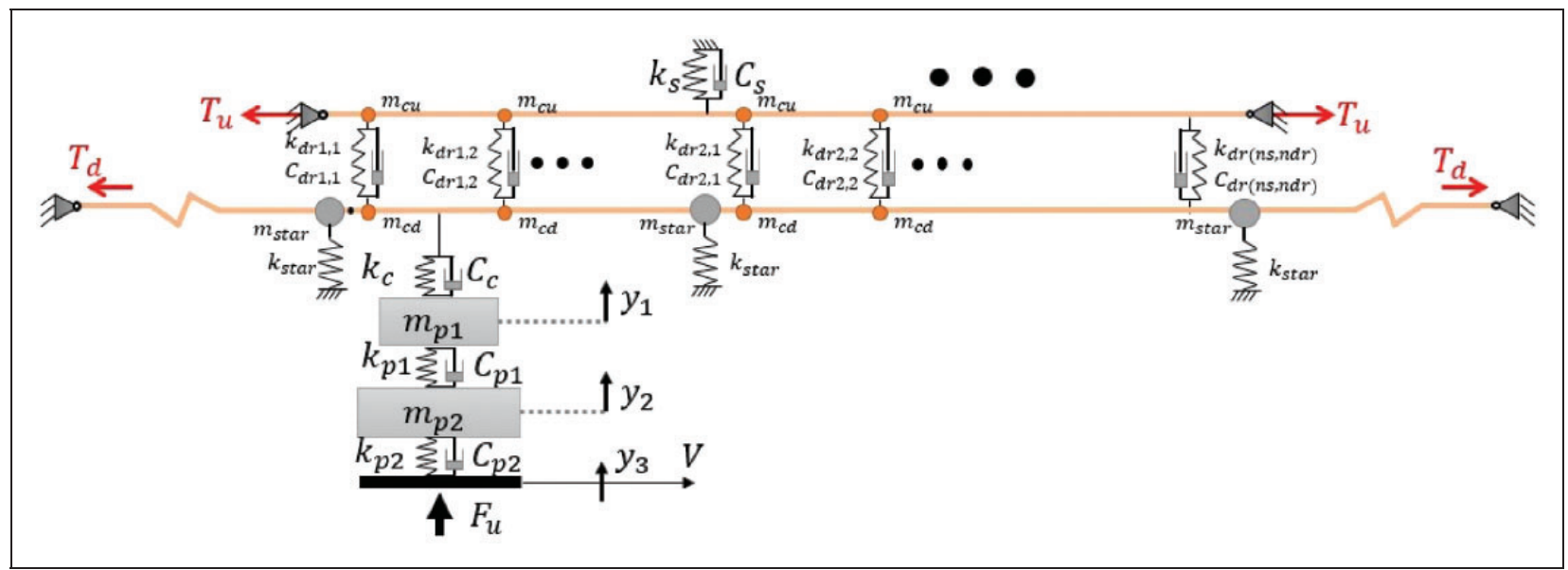

Figure 2. A schematic diagram of the analytical model for the pantograph and catenary system interaction. 
It is possible to convert the partial differential equation into an ordinary differential equation (equation (3)) based on the orthogonality of mode shapes using eigenfunction expansion and inserting equation (2) in equation (1)

$$
w(x, t)=\sum \varphi_{i}(x) q_{i}(t)
$$

In this relation, $\varphi_{i}(x)$ is the $i$ th mode shape, and $q_{i}(t)$ is the $i$ th element of the vector of modal coordinates

$$
\begin{aligned}
& \alpha_{i} \ddot{q}_{i}(t)+\alpha_{i} c_{i} \dot{q}_{i}(t)+\alpha_{i} \omega_{i}{ }^{2} q_{i}(t)= \\
& \quad \times \int_{0}^{l} \varphi_{i}(x)\left(F_{p}(V t, t) \delta(x-V t)+F_{d r}\left(x_{d r}, t\right) \delta\left(x-x_{d r}\right)\right. \\
& \left.\quad+F_{s}\left(x_{s}, t\right) \delta\left(x-x_{s}\right)+F_{c}(t) \delta\left(x-x_{c}\right)\right) \mathrm{d} x, \quad t \geqslant 0
\end{aligned}
$$

$$
\alpha_{i}=\rho A \int_{0}^{l} \varphi_{i}^{2}(x) \mathrm{d} x
$$

Equation (3) is written for the messenger cable and the contact wire. Each element of modal coordinates of wire can be considered as one degree of freedom of that wire. Based on this, if the contact wire is considered having $n$ mode shape and messenger cable considered to have $m$ mode shape, the whole system has $n+m+2$ degrees of freedom considering the pantograph. $M, C$ and $K$ matrices can be achieved, and the entire equation governing the system can be written as equation (5) through integrating contact wire, messenger cable and pantograph equations

$$
M \ddot{Q}+C(t) \dot{Q}+K(t) Q=D F p(t)+E F c(t)
$$

In this matrix equation, $Q$ is a vector of degrees of freedom, which encompasses the modal coordinates of contact wire and messenger cable, and pantograph DOFs. $M$ is the mass matrix of the whole system, which defines the mass of length unit of wires, the mass of clamps of each dropper and mass of pantograph components. Dropper stiffness, natural frequencies of wires, stiffness of pantograph components, and contact stiffness between the pantograph and contact wire are shown in matrix $K$ elements. $C$ expresses the damping proportional to any stiffness of matrix $K$. Since the place of contact between pantograph and contact wire changes, some of the elements of $K$ and $C$ matrices change over time. The elements which determine the relation of contact wire and the first mass of pantographs $\left(m_{p 1}\right.$ in Figure 2$)$ are not constant any more. The uplift force of pantograph and the control force are placed on the left side of the equation and $D$ and $E$ coefficients specify the place to apply the forces.

\section{Controller}

Concerning the analytical model and linearity of the whole system, a linear quadratic optimum controller was used to control the contact wire and pantograph. ${ }^{18}$ A state-space model is required for the design of this type of controller; therefore, by using equations (6) to (9), equation (5) can be converted to a state-space equation

$$
\begin{aligned}
& A=\left[\begin{array}{cc}
0 & I \\
-M^{-1} K & -M^{-1} C
\end{array}\right] \\
& B=\left[\begin{array}{c}
0 \\
M^{-1} D
\end{array}\right] \\
& H=\left[\begin{array}{c}
I \\
M^{-1} E
\end{array}\right] \\
& X(t)=\left[\begin{array}{c}
q(t) \\
\dot{q}(t)
\end{array}\right]
\end{aligned}
$$

The state-space variables (vector $X$ ) are defined as the amount and time derivative of the variables of system DOFs. After obtaining $A, B$ and $H$ values, it is possible to write equations similar to equations (10) and (11) as state-space equations

$$
\begin{aligned}
& \dot{X}(t)=A X(t)+B U(t)+H f(t) \\
& U(t)=G(t) X(t)
\end{aligned}
$$

$U(t)$ is the control input and $f(t)$ is pantograph excitation (uplift force). In the linear control, the control force is assumed as the linear coefficient of statespace variables and controller design is summarised in finding the appropriate $G$ value. To design optimal control, the following integral should be minimised

$$
J=\int_{0}^{t_{f}}\left[X^{T}(t) Q X(t)+U^{T}(t) R U(t)\right] \mathrm{d} t
$$

Matrices $Q$ and $R$ are of paramount importance in controller design. $Q$ determines the importance of state-space variables in controller target. The appropriate selection of this matrix can select controller target or cost function. For instance, the control target can be defined by zeroing the displacement of a point or zeroing the speed of a few meters of the contact wire. This problem, which aims at zeroing the speed of wire overlap point in the middle of overlap span, matrix $\mathrm{Q}$ is defined similar to equation (13)

$$
\begin{gathered}
Q=q_{c}{ }^{T} q_{c} \\
q_{c}=\left[\begin{array}{llllll}
{\left[\varphi_{c}\right]_{1 \times n}} & {[0]_{1 \times m}} & {[0]_{1 \times 2}} & {[0]_{1 \times n}} & {[0]_{1 \times m}} & {[0]_{1 \times 2}}
\end{array}\right]_{1 \times 2(n+m+2)} \\
{\left[\varphi_{c}\right]_{1 \times n}=\left[\begin{array}{llll}
\varphi_{c 1}\left(x_{p t}\right) & \varphi_{c 2}\left(x_{p t}\right) & \ldots & \varphi_{c n}\left(x_{p t}\right)
\end{array}\right]}
\end{gathered}
$$

$q_{c}$ (equation (14)) is the vector whose elements are as many as the state-space variables of the whole system and defines cost function. The arrays of this vector 
consist of six main sections. Mode shapes of contact wire, messenger cable and the displacement of pantograph DOFs form the three initial parts of the vector and their derivation relative to time forms the second three parts of the vector. To make the displacement minimisation of a point on the contact wire be defined as a cost function, vector $\varphi_{c}$ should be located in the first section of the vector $q_{c}$ and other sections become zero. The elements of the vector $\varphi_{c}$ and the mode shapes of contact wire are equal. The $i$ th element of the value of $i$ th mode shape is on the target point.

Matrix $R$ determines a control force in the cost function. In this problem, in which there is only one control force, this matrix is just a number. The smaller the number is, the higher the control force will be, and the better the controller performance will be. $R$ is usually determined with respect to the limitations imposed by the actuator on imposing force. To find the optimal value of $G$ as the controller gain factor, the $S$ value should be found using Riccati equation (equation (16)) and then $G$ value should be found using equation (17)

$$
\dot{S}(t)+S(t) A-\frac{1}{2} S(t) B R^{-1} S(t)+A^{T} S(t)+2 Q=0, S\left(t_{f}\right)=0
$$

$$
G(t)=-\frac{1}{2} R^{-1} B^{T} S(t)
$$

The gain factor was considered fixed and its changes versus time were overlooked in this problem.

\section{The results of using a controller in a simple span}

As stated earlier, one of the reasons to design a controller is to reduce the vertical velocity of the contact wire in the overlap section. A controller has been designed for the governing equations of a simple tensile beam, and the results have been studied to observe the performance of an optimal linear controller. Figure 3(a) shows the model schematic diagram.

It shows that the control force is imposed at the distance of $X_{c}$ from the support and the controller aims at reducing the vertical oscillations within the range of $X_{1}-X_{2}$ from the support (target span). Under the initial conditions of the problem, an initial displacement wave was considered in the middle of the span, and the problem is solved from 0 to $3 \mathrm{~s}$. Table 1 shows the parameters considered for the problem.

Figure 4 shows the effect of the use of the optimal linear controller for the problem of Figure 3(a) on the dynamic response of the whole system.

The displacement wave considered for the initial condition moves towards the two sides of the span. In the side with no controller, the wave hits the support and reflects with reverse amplitude. However, the wave moving where the control force is applied is dissipated by the controller. The controller shows no resistance against the first pass of wave and it traps it between the support and controller connection place. Each time that the wave reflected from the support hits the controller, some of it reflects and the other part is dissipated. The same thing happens to the second wave reflected from the other side of the span after passing under the controller. Figure 4(c) shows that point $P_{t}$ (the point in the middle of the target span) senses only two waves passing under it. The two waves pass through the target span before passing through the controller. This figure shows that the optimal linear controller is capable of managing the traversal waves of a beam under tension in a way that it makes minimum oscillations in the target span.

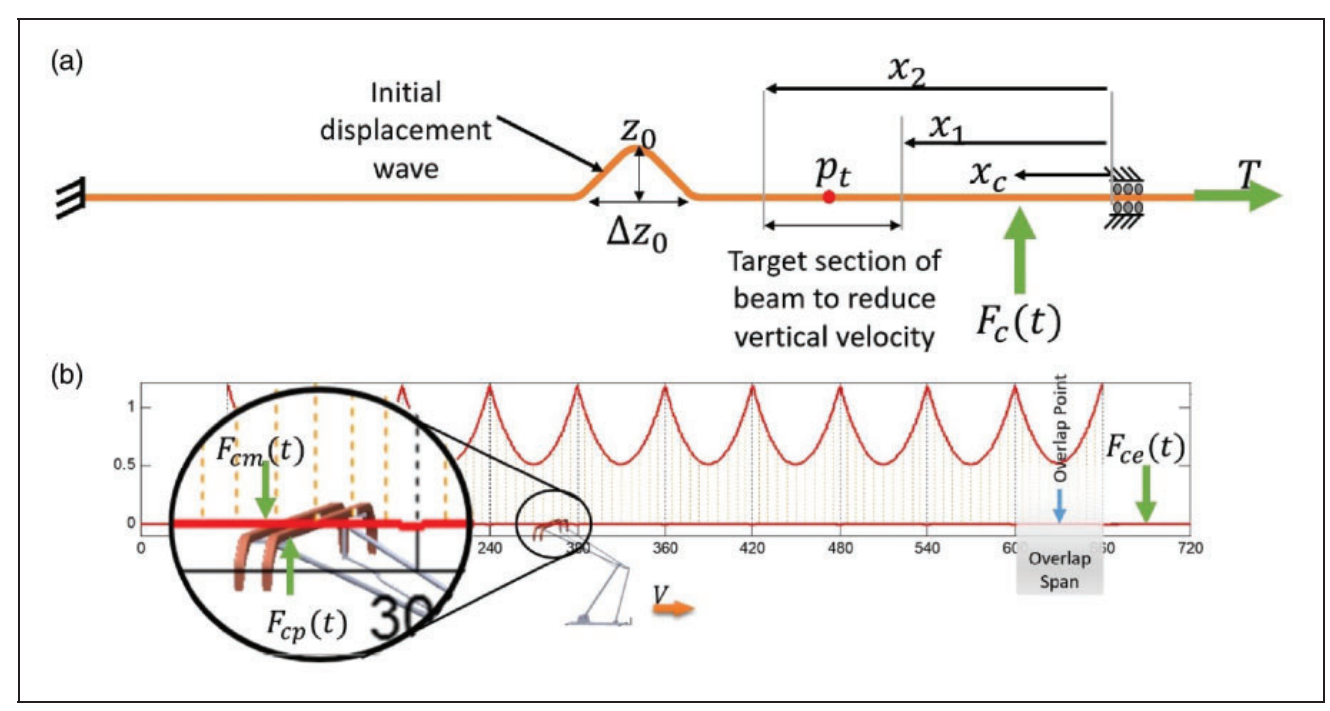

Figure 3. (a) A simple problem to examine the controller performance in a beam under tension and (b) the schematic diagram of the spans and the place to impose control force to control the overlap point. 
Table I. The geometric and dynamic specifications of the tensile beam, catenary and pantograph used in the simulation.

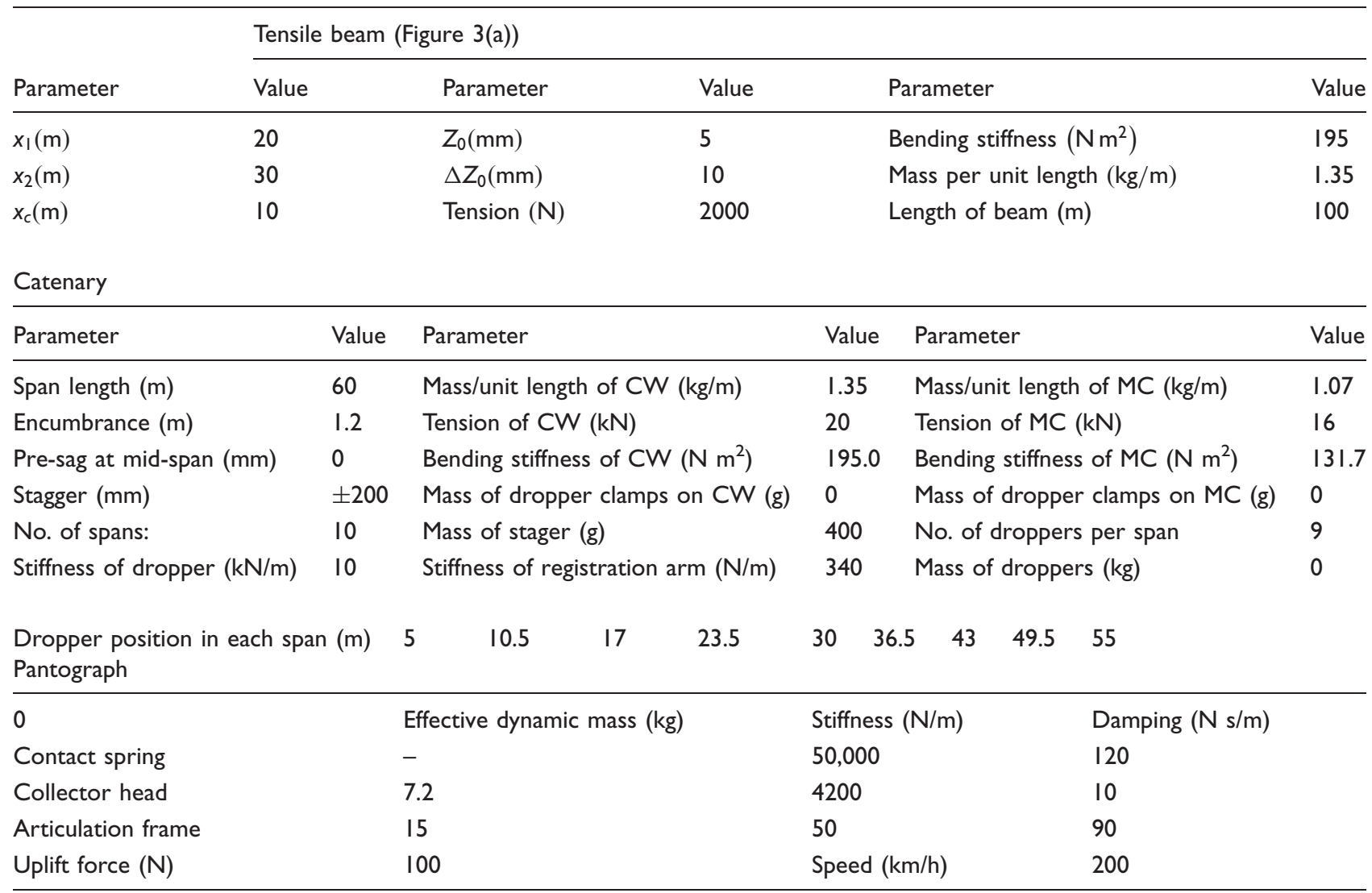

CW: Contact wire; MC: Messenger Cable.
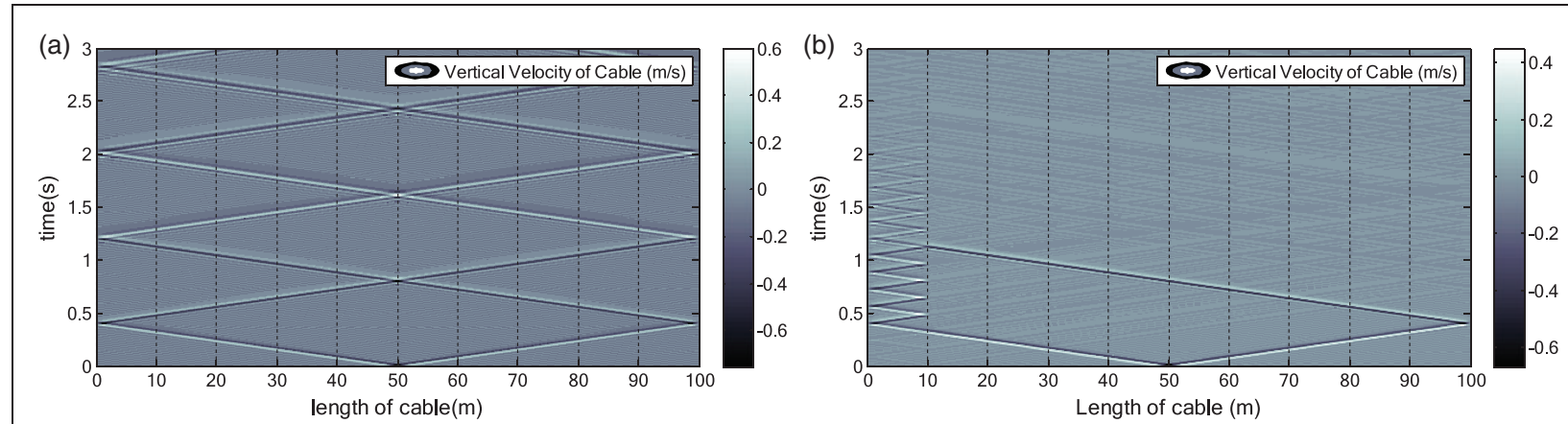

(c)
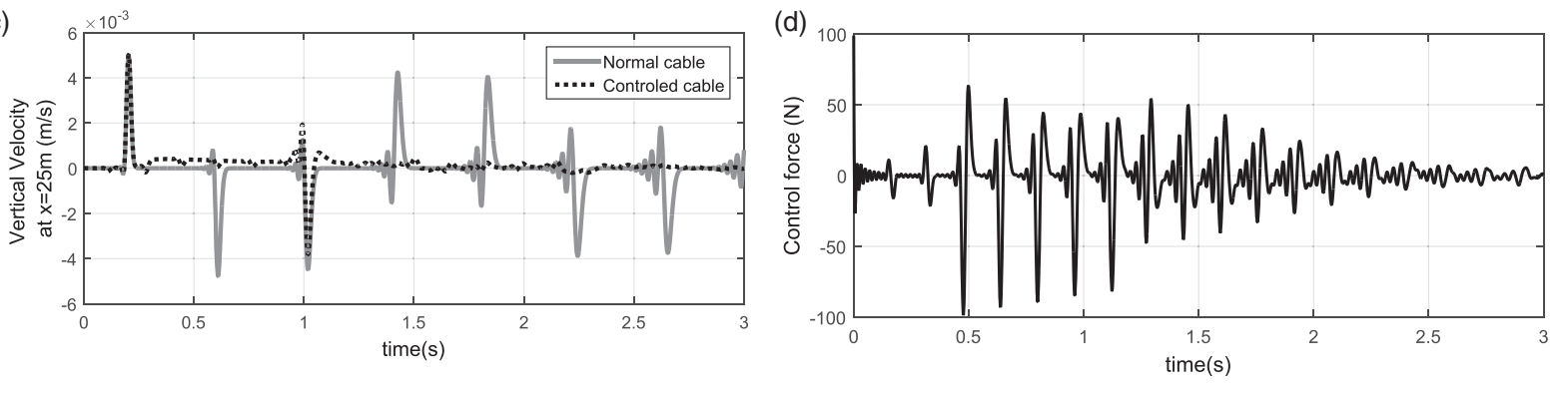

Figure 4. The results obtained from the effect of the controller on a beam under simple tension in the initial excitation of displacement wave (a) contour, position, time, vertical velocity for the beam with no controller; (b) position-time-velocity contour for the beam with the controller; (c) vertical velocity of point $P_{t}$ in Figure 3; and (d) required control force for the controller. 


\section{Different types of controllers on catenary system}

Three types of controllers were considered with two various targets to compare the performance of different controllers (Figure 3(b)). One time, the control force of $F_{c e}(t)$ was considered in the last span of the contact wire near the overlap point of contact wire. Other time, the control force was considered on the contact wire near the contact point with the pantograph $\left(F_{c m}(t)\right)$. Finally, similar to other studies, the control force was imposed on the controller head of pantograph $\left(F_{c p}(t)\right)$. Ten spans of a catenary system were considered in this problem. Table 1 shows the pantograph and catenary system specifications.

In the modal analysis method, the shape of the catenary is described by linear summation of mode shapes and the mode shapes related to higher frequencies have a shorter wavelength. Therefore, it can be concluded that the least number for the modes should be selected in a way that in the distance between the two concentrated loads (or two droppers), more than a pair of nods can be observed in highest mode shape. Such a criterion can be presented as in equation (18)

$$
m \gg \frac{L}{D d r}
$$

In equation (18), $L$ is the whole length of wire or cable, $D d r$ is the minimum distance between two adjacent droppers and $m$ is the required natural frequency to be considered. In this simulation, the first 373 mode shapes of contact wire and the first 310 mode shapes of the messenger wire were considered.

In order to apply a control force to the contact wire near the tension wheel, a linear actuator could be attached to the mass, and by accelerating the mass, the reaction force will be applied to the contact wire as the controlling force. Figure 5(a) shows the schematic diagram of this idea. Applying control force to the contact wire near pantograph would be possible by considering magnetic field near pantograph. Since the contact wire is carrying considerable current near pantograph, it is possible to induct force to a wire with have current (Figure 5(b)). Imposing control force on the pantograph collector head may also be performed by different methods such as an aerodynamic flap, wire, electromagnetic actuator, etc. This study does not aim at examining the source of control force; it mainly focuses on its effect on pantograph interaction and catenary.

As stated earlier, the controller was designed in all three modes using two different attitudes. Initially, attempts were made to maintain the contact force fixed, which is called 'contact force controller' in this study. The controller aims at minimising contact wire impact (related to the following mechanical section) on the pantograph in the overlap section, which is called 'overlap point controller' in this study. In the overlap point controller, the controller aimed at reducing the vertical velocity of contact wire in the overlap point. This study assumed that the pantograph contacts with the contact wire of the following mechanical section from the middle of the 10th span. In the contact force controller, the standard deviation of contact force (contact force SD) is the criterion of controller efficiency; in the overlap point controller, the vertical velocity of the overlap point may indicate controller performance. Figure 6 shows the contact force SD while simulating the problem. The horizontal axis shows the longitudinal position of the contact wire and the vertical axis shows the contact force SD from -30 to $+30 \mathrm{~m}$ at any point. For example, the vertical axis at $\mathrm{X}=30 \mathrm{~m}$ indicates the contact force SD from $X=0 \mathrm{~m}$ to $X=60 \mathrm{~m}$. Therefore, it is clear that it is impossible to define standard deviation for $\mathrm{X}<30 \mathrm{~m}$ and $570 \mathrm{~m}<\mathrm{X}<600 \mathrm{~m}$.

Each diagram of Figure 6 shows the contact force $\mathrm{SD}$ in the three modes. The black line indicates the contact force SD with no controller. The contact force SD in the last spans exceeds other spans with respect to the reflection of the propagated wave. The cost function of contact force oscillations was once considered for controllers (grey line); another time, overlay point velocity oscillations were considered (a grey dashed line). As Figure 6(a) shows, the effect of the

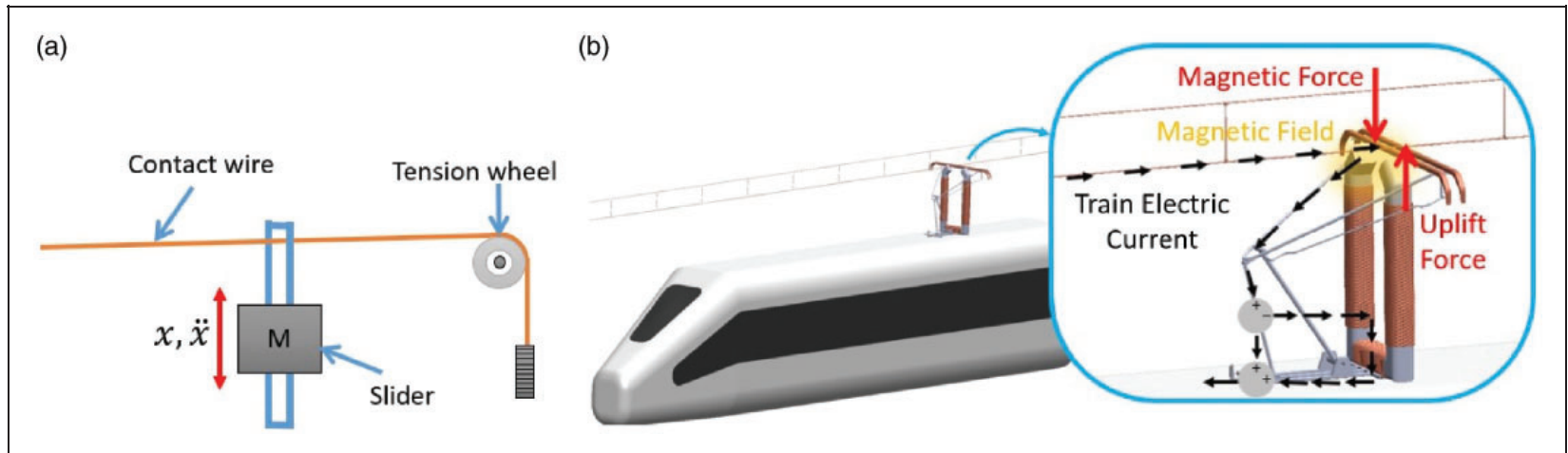

Figure 5. Suggestions on placing the actuator on the contact wire. (a) Last span and (b) contact point vicinity. 


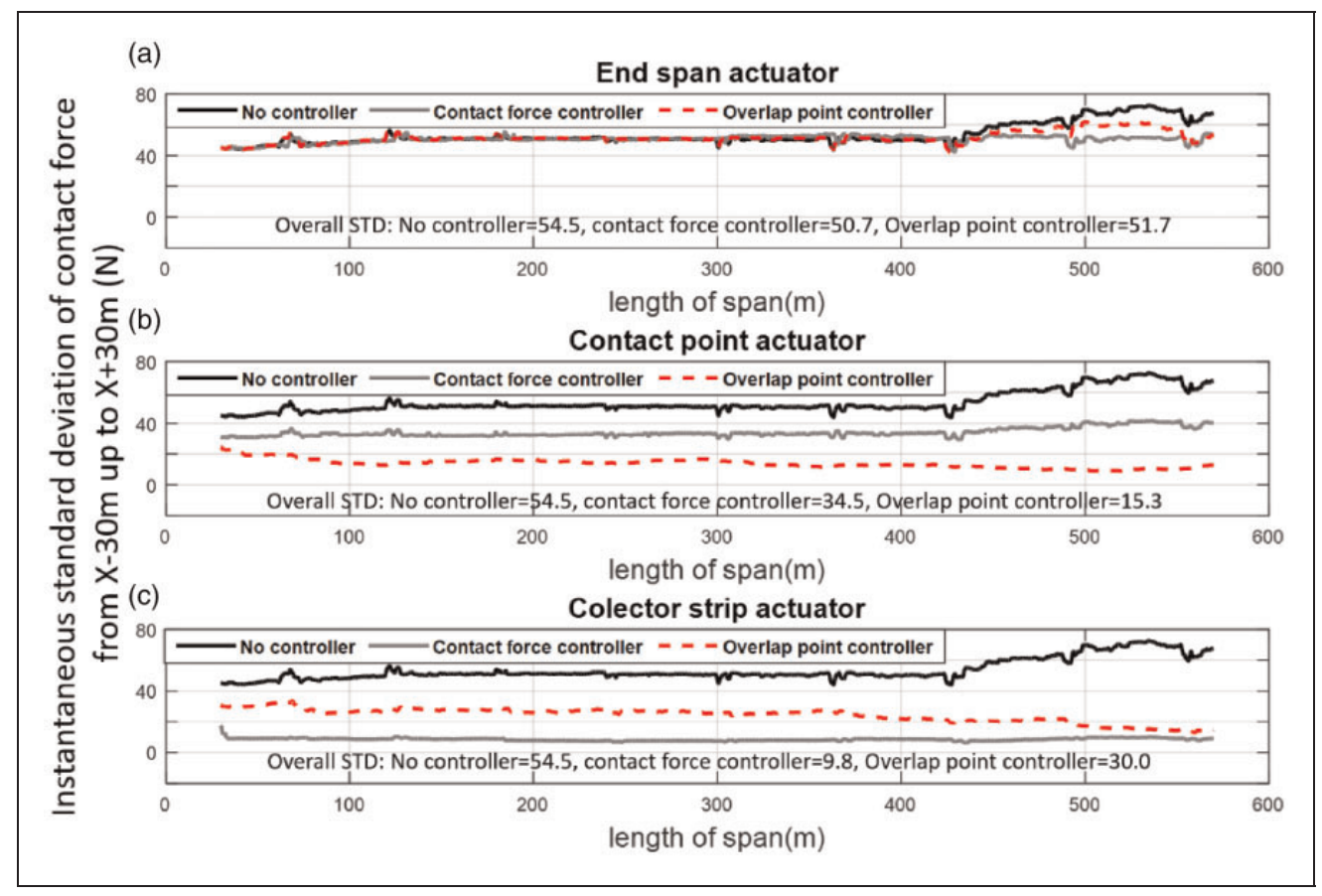

Figure 6. The contact force SD for any designed controllers: (a) actuator in the last span on the contact wire, (b) actuator in the vicinity of the contact point on the contact wire and (c) actuator on the pantograph collector head.

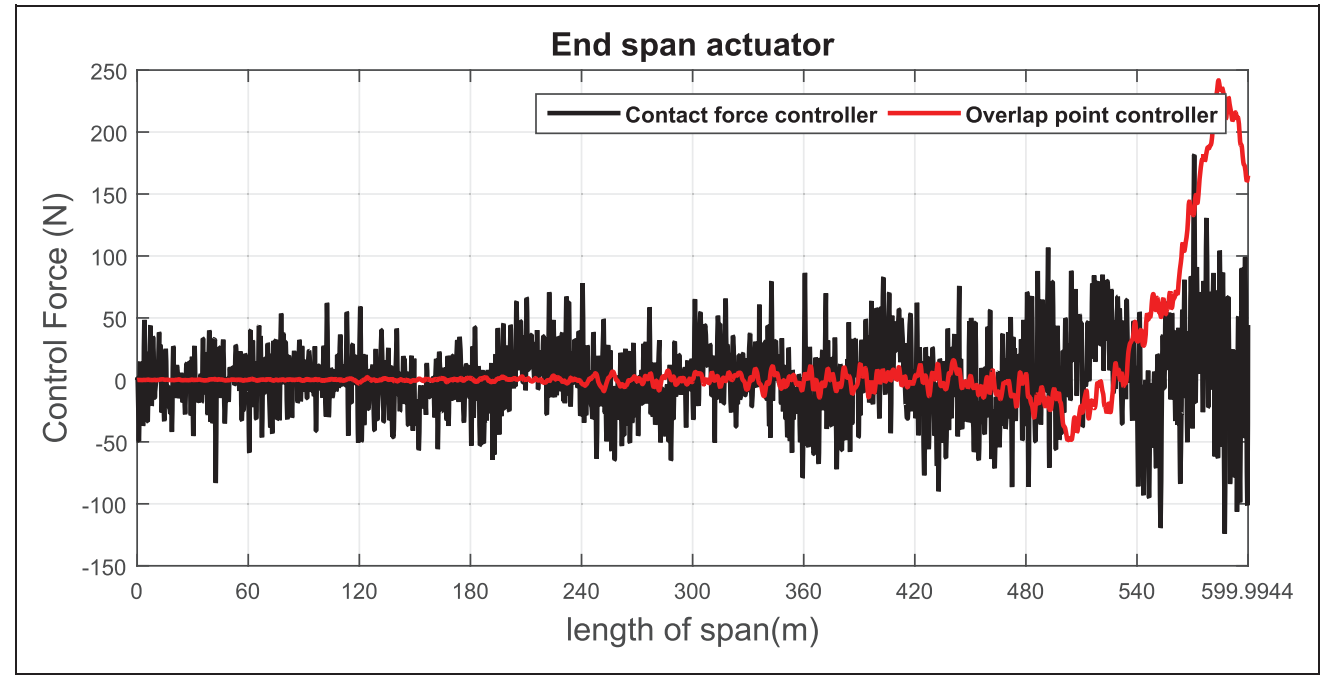

Figure 7. The magnitude of control force for two contact force controllers and time overlap point of the actuator on the last span.

controller is more evident in the last span because the actuator is at the end of the span. When the pantograph is on the first few spans, no wave reaches the overlap point; therefore, as expected, there would be no difference between the mode in which the overlap point controller is on the first spans and the no-controller mode. Contact force oscillations are equal to contact force SD. Therefore, it is predictable that the contact force controller would reduce contact force SD more than the overlap point controller does. Attempts were made in all the six control modes to maintain the maximum control force at about $200 \mathrm{~N}$ through regulating $\mathrm{R}$ in equation (12). For instance,
Figure 7 shows the schematic diagram of the control force required by the controller in the last span.

Figure 7 shows that when the actuator is on the last span and it aims to control overlap point oscillations, the control force is initially negligible; however, when the pantograph approaches the overlap point, it increases rapidly. In Figure 8 the effect of using a controller at end span on the contact force between pantograph and contact wire is illustrated.

As the actuator is located at the end span, the effect of the controller is only observable in last spans. The contact between pantograph and contact wire is modelled with a stiff sparing $\left(K_{c}=50 \mathrm{kN} / \mathrm{m}\right.$ in Figure 2). 


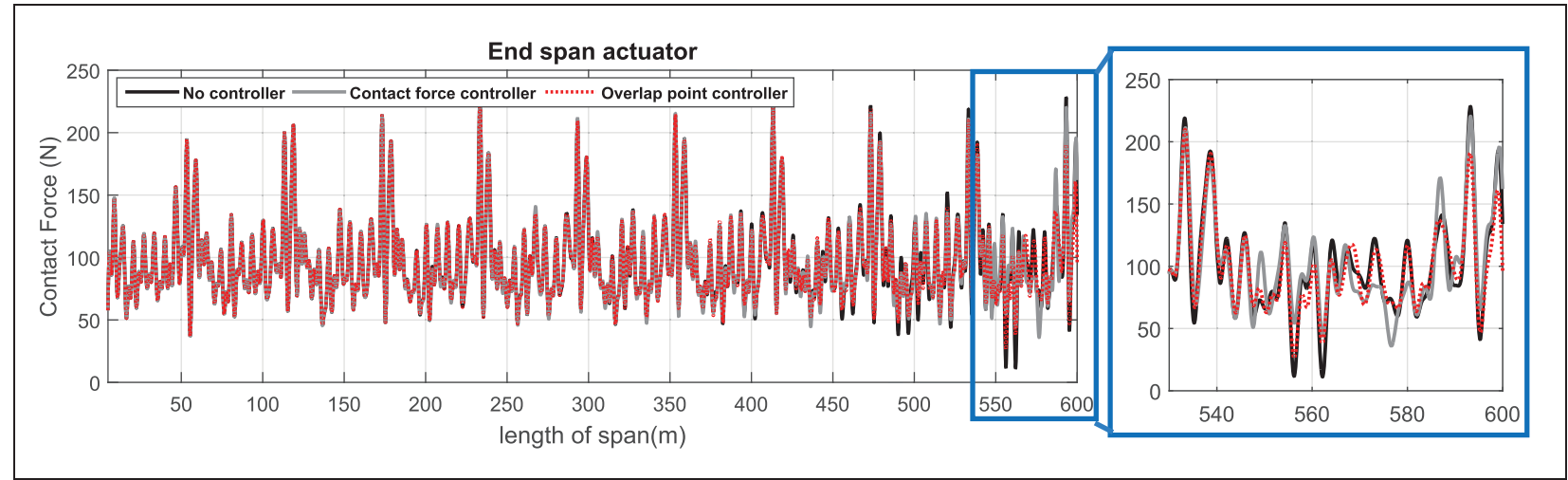

Figure 8. Comparison of the contact without a controller, with contact force controller and overlap point controller. The actuator is located in end span.

Therefore, the amplitude of contact force can be calculated according to equation (19) ${ }^{19,20}$ imposed on one of the variables causing contact force (head of the pantograph displacement). If the control-

$$
F_{c}(t)=\left\{\begin{array}{ll}
K_{c}\left(w_{c}\left(V_{p} t, t\right)-y_{1}\right)+C_{c}\left(\frac{d w\left(V_{p} t, t\right)}{d t}+V_{p}\left(\frac{d w(x, t)}{d x} \mid x=V_{p} t\right)-\dot{y}_{1}\right) & F>0 \\
0 & F \leqslant 0
\end{array}\right\}
$$

In this equation, $F_{c}$ is the contact force; $K_{c}$ and $C_{c}$ are the stiffness and damping of contact point, respectively; $w_{c}$ and $y_{1}$ are the deflections of contact wire and displacement of collector head of pantograph as well.

In Figure 6(b), the actuator was applied near the contact point and on the contact wire. In this mode, despite the earlier mode, both controllers were able to make a considerable reduction in the contact force oscillations. Interestingly, the overlap point controller outperformed in reducing oscillations. The contact force is a function of the relative displacement between the head of pantograph and contact wire at the contact point. In other words, the contact force is affected by contact wire displacement and head of the pantograph. If the whole system is divided into pantograph and catenary sections, controller performance will be more favourable when both the cost function and the actuator are located in the same section. In Figure 6(b) the actuator is located in the contact point in the catenary. The overlap point controller is located on the contact wire as well, while for contact force controller, one parameter which describes contact force (equation (19)) is a deflection of contact wire which belongs to catenary and the other parameter is vertical displacement (or velocity) of collector head, which belongs to the pantograph. Therefore, it can be expected that for the contact point actuator, the overlap point controller has better performance than contact force controller.

In the last mode (Figure 6(c)), it is assumed that the actuator is on the pantograph collector and it imposes the control force on the head of the pantograph directly. In this mode, the control force is immediately ler aims at reducing contact force oscillations, it may prevent to change the distance of head of pantograph and contact wire and it may reduce contact force SD considerably. Overlap point controller could reduce contact force SD. In this controller, the controller effect becomes more tangible with the pantograph approaching the overlap point.

As mentioned earlier, two separate targets were considered in the simulations, and Figure 6 shows only the controller effect on one of the targets (reduction of contact force oscillations). Figure 9 shows the performance of controllers to achieve another target.

Since the pantograph touch the second contact wire at point $\mathrm{x}=570 \mathrm{~m}$, this point has been zoomed in the diagrams. Figure 6 shows that even if it aims to reduce overlap point oscillations, contact quality improvement can be guaranteed as one of the side advantages of the controller. This is due to the creation of damping in the contact wire and prevention of the return of wave from the supports. However, Figure 9 proves that the opposite is not true. In other words, when the controller focuses on contact quality improvement, this will not reduce the overlap point oscillations of the contact wire. Figure 9(a) shows that if the control actuator is on the last span and it is aimed to reduce overlap point oscillations, the vertical velocity of the point will generally reduce. However, when the vertical pantograph is crossing, the overlap point is similar to the time when no controller is applied. If the contact force controller is used, overlap point velocity oscillations will remain from the beginning of the simulation, as the actuator is near the overlap point and it affects its vertical velocity considerably. 


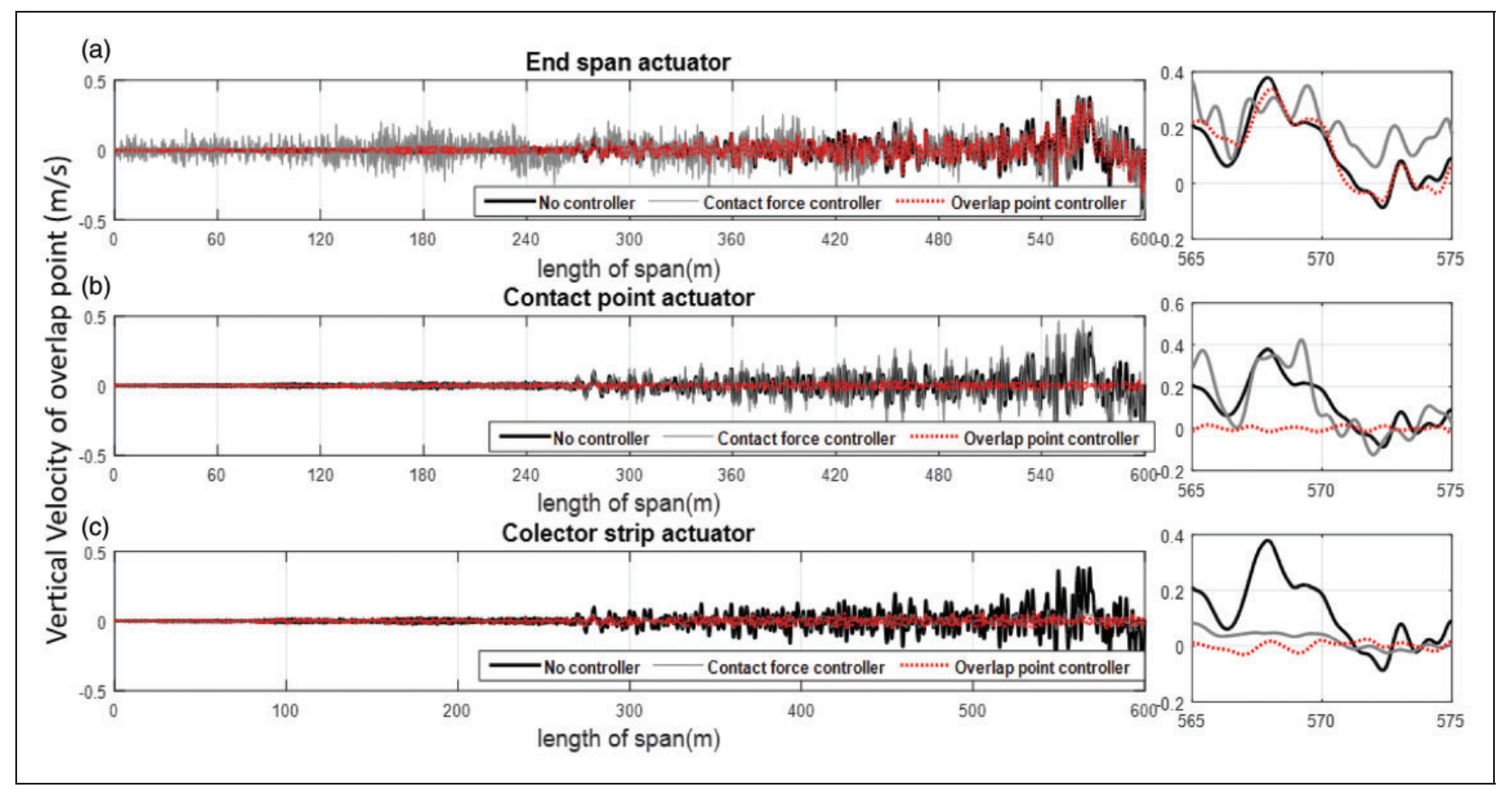

Figure 9. Performance of controllers in reducing the velocity of the pantograph to hit the following contact wire in the overlap point: (a) the actuator in the last span on the contact wire, (b) the actuator near the contact point on the contact wire and (c) the actuator on the pantograph collector head.

Table 2. A summary of the results obtained from different control actuators with varied control targets.

\begin{tabular}{|c|c|c|c|c|c|}
\hline Location of actuator & Aim of controller & $\begin{array}{l}\text { Standard deviation } \\
\text { of overlap } \\
\text { point's vertical } \\
\text { velocity }(\mathrm{m} / \mathrm{s})\end{array}$ & $\begin{array}{l}\text { Overlap point } \\
\text { vertical velocity } \\
\text { while pantograph } \\
\text { is passing }(\mathrm{m} / \mathrm{s})\end{array}$ & $\begin{array}{l}\text { Standard deviation } \\
\text { of the contact } \\
\text { force }(N)\end{array}$ & $\begin{array}{l}\text { Mean value } \\
\text { of contact } \\
\text { force }(N)\end{array}$ \\
\hline \multirow[t]{2}{*}{ Last span } & Contact force & 0.091 & 0.19 & 50.73 & 91.04 \\
\hline & Velocity of overlap point & 0.057 & 0.21 & 51.68 & 90.65 \\
\hline \multirow[t]{2}{*}{ Contact point } & Contact force & 0.087 & 0.06 & 34.51 & 79.93 \\
\hline & Velocity of overlap point & 0.010 & -0.01 & 15.30 & 112.62 \\
\hline \multirow[t]{2}{*}{ Collector head } & Contact force & 0.019 & 0.04 & 9.76 & 25.37 \\
\hline & Velocity of overlap point & 0.015 & 0.02 & 30.01 & 35.62 \\
\hline No controller & & 0.067 & 0.18 & 54.50 & 91.22 \\
\hline
\end{tabular}

Figure 9(b) shows that the overlap point velocity will reduce if the actuator is on the contact wire and the overlap point controller is used. However, in case it aims to improve the contact quality, it will not have a noticeable effect on reducing overlap point velocity. Figure 9(c) shows that when the actuator is on the pantograph collector head, both the overlap point controller and contact force controller may reduce overlap point oscillations. In this mode, the mean value of the contact force reduces considerably. In other words, when the actuator is placed on the collector's head, the controller reduces the mean of contact force to lower contact force oscillations, and it increases the probability of detachment of pantograph from the contact wire. Table 2 shows a summary of the efficiency of each controller.
It is expected that the controller, which was designed aiming at improving contact quality, would further reduce contact force SD. However, the controller used to reduce overlap point oscillations may not necessarily lead to the reduction of the vertical velocity of the overlap point when the pantograph is crossing. In other words, the vertical velocity of the overlap point is a vector in which the controller attempts to reduce all its values. However, the overlap point vertical velocity during the cross of pantograph is only one point of the vector whose value does not necessarily reduce as compared with the no-controller mode. Table 2 shows that if the actuator is on the last span, the overlap point velocity will increase at the time of crossing in both the contact force controller and the controller of overlap point velocity. However, 
the overlap point controller was able to reduce the SD of overlap point velocity by about $20 \%$.

Meanwhile, the overlap point controller has a very favourable performance when the actuator is near the contact point. The controller could reduce the contact force SD from 54 to $15 \mathrm{~N}$. On the other hand, it reduced the overlap point velocity considerably, as the velocity reduced from 18 to $1 \mathrm{~cm} / \mathrm{s}$ at the time of pantograph crossing. All the improvements were made while the average contact force was not reduced and it was increased slightly $(112 \mathrm{~N}$ of average contact force was obtained from $100 \mathrm{~N}$ uplift force). This is the highest superiority of contact point actuator to the actuator on the pantograph collector head. If the actuator is on the pantograph head collector, it reduces its average value to reduce contact force oscillations. Table 2 shows that although the actuator installed on the controller's head with contact force controller could reduce contact force SD and overlap point velocity considerably, it reduced the average contact force and it created $25 \mathrm{~N}$ of the average contact force from the uplift force of $100 \mathrm{~N}$. As the results of Table 2 show, among the selected actuators and the two cost functions, the use of the actuator at the contact point and the cost function of overlap point velocity will lead to the most favourable result.

\section{Conclusions}

This report considered an analytical model for the interaction of the pantograph and the catenary system of an electric fleet. To prevent pantograph wear and to improve contact quality, despite other studies, two types of novel controllers were designed for the catenary system, and the results were compared with the time the controller is placed on the controller head of pantograph. Regarding high tensile load in catenary cables and small deflection of catenary due to pantograph motion, most of the studies in this field consider linear equation for catenary and pantograph motion. Performance of the designed linear quadratic regulator was first examined on a tensile beam, and the results showed that the controller might trap and dissipate the propagated wave. The controller was then added to complete the catenary system model with three different actuators including collector head actuator, last span actuator of contact wire and contact point actuator on contact wire. Two cost functions were considered for any actuator. Contact quality improvement was the traditional objective of all the studies in this field. In addition to contact quality, elimination of the overlap point of the contact wire was considered as the other objective of the study, as most studies discuss the overlap point of contact wire as the bottleneck of pantograph contact quality and contact wire. The results for the simulation of the designed linear optimal controller show that if the controller is designed to reduce overlap point oscillations, it will improve contact quality, as it dissipates the wave returning from the support. However, if the controller aims at improving contact quality, it will not necessarily reduce the vertical velocity of the overlap point. The simulation results showed that the actuators installed on the collector's head and the contact point outperformed the last span actuator; however, if the actuator is installed on the collector, it reduces the average contact force, which may lead to pantograph de-wirement. Finally, it can be mentioned that the use of the actuator on the contact wire near the contact point with the pantograph with the cost function of overlap point oscillations may reduce the overlap point velocity considerably and improve contact quality.

\section{Acknowledgements}

The authors would like to acknowledge Mr Mohrich Joerg from Balfour Beatty Rail Company and Prof. Giorgio Diana from Polytechnic University of Milan for sharing experiences and presenting challenges.

\section{Declaration of Conflicting Interests}

The author(s) declared no potential conflicts of interest with respect to the research, authorship, and/or publication of this article.

\section{Funding}

The author(s) disclosed receipt of the following financial support for the research, authorship, and/or publication of this article: This research was supported by the office for 'National Master Plan for High-Speed Trains' at the Iran University of Science and Technology.

\section{ORCID iD}

Habibollah Molatefi (D) http://orcid.org/0000-0002-75849637

\section{References}

1. Ambrósio J, Pombo J, Antunes P, et al. PantoCat statement of method. Vehicle Syst Dyn 2015; 53: 314-328.

2. Cho YH. SPOPS statement of methods. Vehicle Syst Dyn 2015; 53: 329-340.

3. Collina A and Bruni S. Numerical simulation of pantograph-overhead equipment interaction. Vehicle Syst Dyn 2002; 38: 261-291.

4. Finner L, Poetsch G, Sarnes B, et al. Program for catenary-pantograph analysis, PrOSA statement of methods and validation according EN 50318. Vehicle Syst Dyn 2015; 53: 305-313.

5. Seo J-H, Sugiyama H and Shabana AA. Three-dimensional large deformation analysis of the multibody pantograph/catenary systems. Nonlinear Dyn 2005; 42: 199-215.

6. Ambrósio J, Pombo J and Pereira M. Optimization of high-speed railway pantographs for improving pantograph-catenary contact. Theor Appl Mech Lett 2013; 3: 50-56.

7. Pombo J and Ambrosio J. Influence of pantograph suspension characteristics on the contact quality with the catenary for high speed trains. Comput Struct 2012; 110: $32-42$. 
8. Song Y, Ouyang H, Liu Z, et al. Active control of contact force for high-speed railway pantograph-catenary based on multi-body pantograph model. Mech Mach Theory 2017; 115: 35-59.

9. Ko MT. "Robust control of the contact force of an active pantograph for high speed trains," Niigata University, 2017.

10. Balestrino A, Bruno O, Landi A, et al. Innovative solutions for overhead catenary-pantograph system: wire actuated control and observed contact force. Vehicle Syst Dyn 2000; 33: 69-89.

11. O'Connor D, Eppinger S, Seering W, et al. Active control of a high-speed pantograph. J Dyn Syst Meas Control 1997; 119: 1-4.

12. Pappalardo CM, Patel MD, Tinsley B, et al. Contact force control in multibody pantograph/catenary systems. Proc IMechE, Part K: J Multi-body Dynamics 2016; 230: 307-328.

13. Bruni S, Bucca G, Carnevale M, et al. Pantographcatenary interaction: recent achievements and future research challenges. Int J Rail Transp 2018; 6: 57-82.

14. Harell P, Drugge L and Reijm M. Study of critical sections in catenary systems during multiple pantograph operation. Proc IMechE, Part F: J Rail and Rapid Transit 2005; 219: 203-211.

15. Kiessling F, Puschmann R, Schmieder A, et al. Contact lines for electric railways: planning, design, implementation, maintenance. Erlangen, Germany: John Wiley \& Sons, 2018.

16. Bruni $\mathrm{S}$, et al. The results of the pantograph-catenary interaction benchmark. Vehicle Syst Dyn 2014; 53(3): $1-24$.

17. Kia SH, Bartolini F, Mpanda-Mabwe A, et al. Pantograph-catenary interaction model comparison. In: IECON 2010-36th annual conference on IEEE industrial electronics society, Glendale, AZ, 2010, pp.15841589. IEEE.

18. Soong T. State-of-the-art review: active structural control in civil engineering. Eng Struct 1988; 10: 74-84.

19. Abdullah MA, Michitsuji Y, Nagai M, et al. Integrated simulation between flexible body of catenary and active control pantograph for contact force variation control. J Mech Syst Transp Logist 2010; 3: 166-177.

20. Kulkarni S, Pappalardo CM and Shabana AA. Pantograph/catenary contact formulations. $J$ Vib Acoust 2017; 139: 011010.

\section{Appendix}

\section{Notation}

$c_{i} \quad$ damping of the $i$ th mode shape

$c_{p 1}$
$C_{c}$

$C_{d r i, j}$

$C_{s}$

EI

$F_{c}$

$F_{c e}$

$F_{c m}$

$F_{c p}$

$F_{d r}$

$F_{s}$

$F_{u}$

$k_{d r i, j}$

$k_{p 1}$

$k_{p 2}$

$k_{\text {star }}$

$K_{c}$

$K_{s}$

$m_{c d}$

$m_{c u}$

$m_{p 1}$

$m_{p 2}$

$m_{\text {star }}$

$T_{d}$

$T_{u}$

$w(x, t)$

$x_{c}$

$x_{d r}$

$x_{s}$

$y_{1}$

$y_{2}$

$\rho A$

$\varphi_{i}(x)$

$\omega_{i}$ damping articulation frame of the pantograph

damping of the contact point

damping of the $j$ th dropper in the $i$ th

span

damping of support at masts

bending stiffness

controlling force

end span controlling force

contact point controlling force

collector head controlling force

dropper force

support force

uplift force of the pantograph

stiffness of $j$ th dropper in the $i$ th span stiffness of the collector head of the pantograph

stiffness articulation frame of the pantograph

stiffness of the steady arm

stiffness of the contact point stiffness of the support at masts mass of the dropper clamps on the contact wire

mass of the dropper clamps on the messenger cable

mass of the collector head of the pantograph

mass of the articulation frame of the pantograph

equivalent mass of the steady arm on the contact wire tension of the contact wire tension of the messenger cable vertical deflection of the wire location of the controlling force location of the dropper location of support at masts vertical displacement of the collector head of the pantograph vertical displacement of the articulation frame of the pantograph

mass per unit length of the wire $i$ th mode shape of the wire $i$ th natural frequency of the wire 